\title{
Pseudo-Univariate Calibration as an Analytical Tool to Determine Antioxidant Activity: An Alternative to DPPH Method Applied to the Evaluation from Extracts of Turmeric Powder
}

\author{
Mirian S. Laczkowski ${ }^{1}$, Thays R. Gonçalves ${ }^{1}$, Sandra T. M. Gomes ${ }^{1}$, Paulo Henrique Março², \\ Patrícia Valderrama $^{2}$ (D), Makoto Matsushita ${ }^{1}$ \\ ${ }^{1}$ Universidade Estadual de Maringá - UEM. 87020-900, Maringá, PR, Brazil \\ ${ }^{2}$ Universidade Tecnológica Federal do Paraná - UTFPR. P.O. Box 271, 87301-899, Campo Mourão, PR, Brazil
}

A pseudo-univariate calibration model was proposed as an alternative method to determine the antioxidants activity (\%RSA) in extract from turmeric powder by using the relative intensity from multivariate curve resolution with alternating least squares (MCR-ALS) from near-infrared (NIR) spectra. An extraction was performed by using an ultrasound with probe, ethanol $70 \%$ and time 15 minutes. For pseudo-univariate calibration, eight independent samples were prepared from the curcuminoid extracts. NIR spectra were collect from 900 to $1700 \mathrm{~nm}$, and to these samples the percentage of inhibition of DPPH was determined with reference method. The signal for antioxidants was isolated from the signal to the other analytes in the sample by applying MCR-ALS to the NIR spectra. The relative intensity profile related to the antioxidants was employed in a pseudo-univariate calibration against the \%RSA obtained by the reference conventional method based on DPPH. The results of the \%RSA determination in four independent samples with mean \%RSA (obtained by the reference method) of $90.26 \%$ show a mean $\%$ RSA of $87.5 \%$, showing the possibility of \%RSA determination directly from NIR spectra of turmeric powder extracts by using the multivariate resolution method MCR-ALS.

Keywords: MCR-ALS; pseudo-univariate calibration; NIR; DPPH; antioxidant activity.

\section{INTRODUCTION}

Analytical chemistry begins to reach a higher degree of maturation after the introduction of chemometrics. At the time it was hoped that chemometrics could change the way in which analytical methods were developed [1]. It is now well known that chemometrics is increasingly present in analytical methodologies, especially those involving spectroscopies. However, many methodologies, especially in food analysis, still deserve an update. An example is an evaluation of antioxidants through the 2,2-Diphenyl-1-picrylhydrazyl (DPPH) method.

There is no consensus on the scientific literature about the DPPH method. In each case the method is optimized in some different senses. Recently, in a previous study, it was proposed to evaluate the antioxidant activity by using near-infrared (NIR) spectroscopy [2]. In this sense, the main objective of this work was to perform a pseudo univariate calibration based on NIR spectroscopy coupled with multivariate curve resolution with alternating least squares (MCR-ALS) for determination of the antioxidant activity from extracts of turmeric powder.

Some phenolic compounds can form complexes with metals, inhibit lipoxygenase and capture free radicals, such as DPPH [3]. DPPH is known to be characterized as a stable organic free radical, being used in more than $90 \%$ in the studies of evaluation of antioxidants in matrices of pure substances, mixtures or complexes mixtures [4-6]. However, this conventional method presents several drawbacks, as the use of significant amounts of reagents, standards, samples, and time. In this way, when coupled 
with instrumental techniques, chemometric tools of multivariate analysis can be an update to the results interpretation, allowing a more complete and efficient understanding of the analytical results $[7,8]$.

Curve resolution is a chemometric tool that has been employed for pseudo univariate calibration $[2,9,10]$. This tool promotes the separation of the analyte signals without the need for physical separations [11]. MCR-ALS is a curve resolution chemometric tool that aims to resolve signal mixtures by recovering pure spectra and its relative intensity for the analytes in a sample [11-13]. Then, this chemometric tool can be applied for quantification when that relative intensity profile for the analyte of interest is employed in the construction of a pseudo univariate calibration curve [11].

\section{MATERIAL AND METHODS}

Fresh turmeric rhizomes were obtained in Maringá, Paraná, Brazil and harvested between August and November 2016/2017. The turmeric rhizomes were washed, sliced $(0.50$ to $0.70 \mathrm{~mm})$ and submitted to drying in an oven with air circulation at $40 \pm 0.5^{\circ} \mathrm{C}$ for 36 hours, this method was adapted from Vieira \& Jorge [14]. After drying the rhizomes were ground in a knife mill, being standardized granulometry (120 mesh), obtaining the rhizomes in powder.

The extraction from turmeric powder was based on preliminaries studies [15-17], by using $0.1 \mathrm{~g}$ of powder rhizomes and $10 \mathrm{~mL}$ of ethanol $70 \%$ in an Ultrasound (Fisher Scientific - FB120 - 2000, Park Lana - Pittsburg, PA - 15275) with probe (Fisher Scientific - Model E18), 120 W of power and frequency $100 \%(20 \mathrm{kHz})$ in room temperature $\left(25^{\circ} \mathrm{C}\right), \mathrm{pH} 5.5$, and time of 15 minutes. The obtained extract was centrifuged for 20 minutes at $4000 \mathrm{rpm}$.

In order to verify the extraction, the curcuminoids concentration $\left(\mathrm{g} \mathrm{mL}^{-1}\right)$ was evaluated. A standard calibration curve was obtained (with ethanol $70 \%$ ) based on the curcumin standard (65\% of curcumin, $35 \%$ other curcuminoids, Sigma Aldrich) in the range from $8.10^{-6}$ to $8.10^{-5} \mathrm{~g} \mathrm{~mL}^{-1}$, by using the absorption at $427 \mathrm{~nm}$ (Ocean Optics Spectrophotometer Red Tide, USB650 UV model).

The antioxidant activity from the extract was determined by the DPPH method employing the reagent 2,2-Diphenyl-1-picrylhydrazyl (Sigma Aldrich). DPPH solution in ethanol was prepared in the concentration of $28 \mathrm{mg} \mathrm{mL}^{-1}$. This concentration was used in previous studies in order to show absorbance at $515 \mathrm{~nm}$ between 0.6 and $0.7 \mathrm{u}$.a [2,18,19].

The determinations were performed (in triplicates) with $100 \mu \mathrm{L}$ of the extract and $3900 \mu \mathrm{L}$ of the DPPH solution. A control sample was prepared with $100 \mu \mathrm{L}$ of ethanol and $3900 \mu \mathrm{L}$ of DPPH solution. Each mixture was placed in the dark for 30 minutes. The absorption was measured in a spectrophotometer (Ocean Optics Spectrophotometer Red Tide, USB650 UV model) at $515 \mathrm{~nm}$. The antioxidant activity (\%RSA) was calculated as the percentage of inhibition of the DPPH radical through equation 1 [20].

$$
\% \operatorname{RSA}=\left[\left(A_{\text {control }}-A_{\text {sample }}\right) / A_{\text {control }}\right] \times 100
$$

where: $A_{\text {control }}$ is the absorbance at $515 \mathrm{~nm}$ for the control sample and $A_{\text {sample }}$ is the absorbance at 515 $\mathrm{nm}$ for the samples.

For pseudo-univariate calibration, eight independent samples were prepared from the curcuminoid extracts by adding 25, 50,75, 100 and $150 \mu \mathrm{L}$ of extract in $2 \mathrm{~mL}$ of distilled water. NIR spectra were collect from 900 to $1700 \mathrm{~nm}$ (MicroNIR - JDSU) with a glass cuvette. To these samples, the percentage of inhibition of DPPH was determined at the same time, by using the previously cited conventional method.

The signal for antioxidants was isolated from the signal to the other analytes in the sample by applying MCR-ALS to the NIR spectra. A graphical user-friendly interface for MCR-ALS [21], for Matlab software (R2007b) was employed.

The MCR-ALS provided a spectral profile for each constituent in the sample and its relative intensity profile. The relative intensity profile related to the antioxidants was employed in a pseudo-univariate 
calibration against the \%RSA obtained by the reference conventional method based on DPPH.

\section{RESULTS AND DISCUSSION}

The curcuminoids concentration for the extracts was $0.718 \mathrm{mg} \mathrm{L}^{-1}$. The NIR spectra of the antioxidant extracts from turmeric rhizomes are shown in Figure 1. The spectral baseline was corrected by savgol algorithm [22], through 7 points, first order polynomial and first derivative.
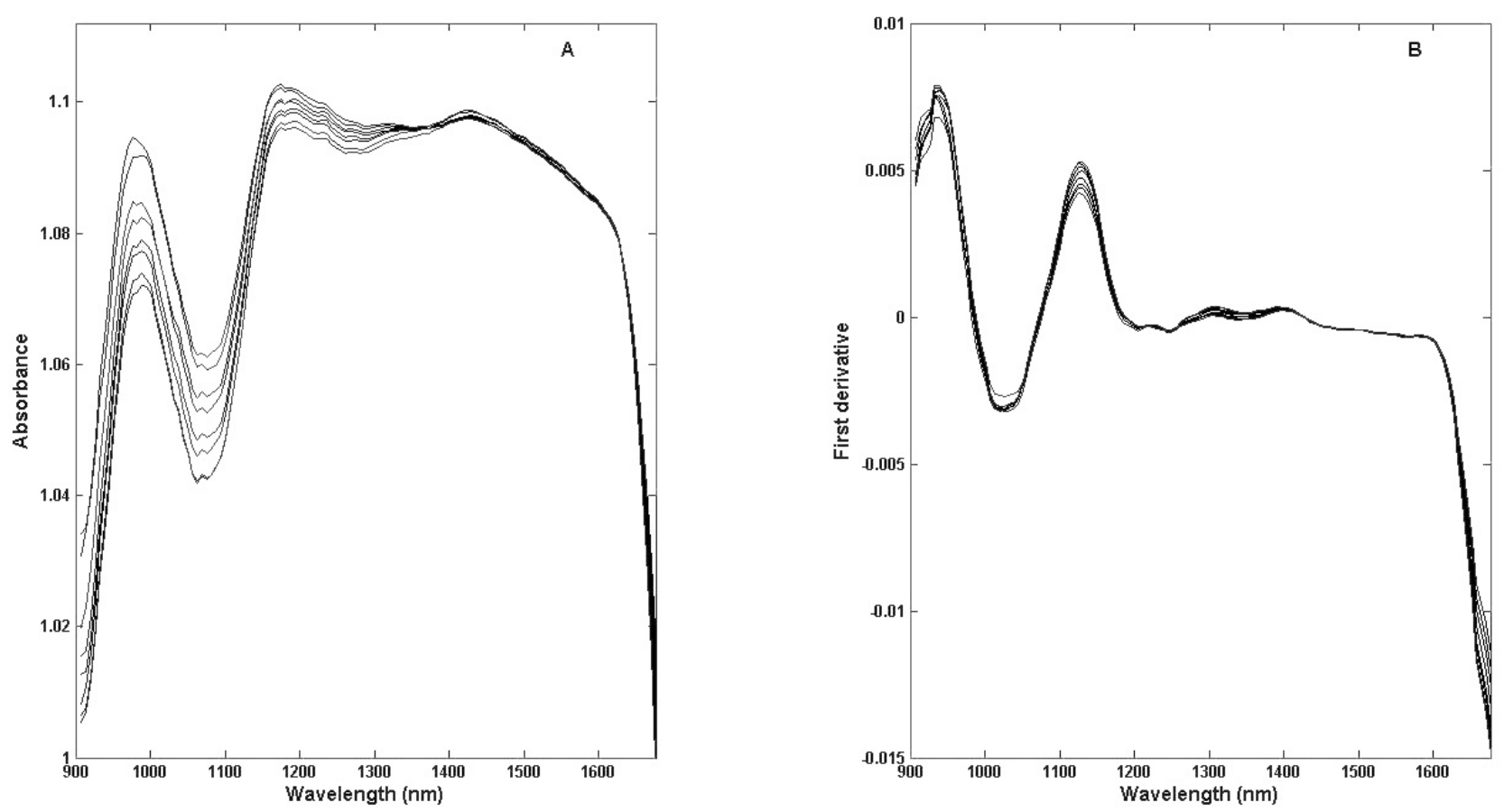

Figure 1. NIR spectra of antioxidant extracts from turmeric powder. (A) Raw spectra. (B) Spectra after baseline correction.

For the spectra after baseline correction, a mathematical rank was estimated from the percentage of variance explained by the Principal Component Analysis (PCA) [9]. The rank equal to four was considered since the first four principal components retain $99.40 \%$ variance from this data set.

MCR-ALS was applied to resolve the pure spectra and concentration profiles for four different components in antioxidant extracts from turmeric powder. During alternating least squares optimization, no constraints were employed. The initial estimates for $\mathbf{S}^{\top}$ were achieved by the PCA loadings [23]. Figure 2 shows the concentration profiles and recovered spectra resolved for antioxidant extracts. The relative intensity profile related to the antioxidants (bold blue solid line) shows a \%RSA decreasing with the antioxidant concentration increasing. The absorptions for the recovered spectra related to the antioxidants are attributed for the second and third overtones of $\mathrm{CH}_{3}, \mathrm{CH}_{2}$, and $\mathrm{CH}$, besides the third overtone absorption of $\mathrm{R}-\mathrm{OH}, \mathrm{Ar}-\mathrm{OH}$ and $\mathrm{Ar}-\mathrm{CH}$ [24]. 

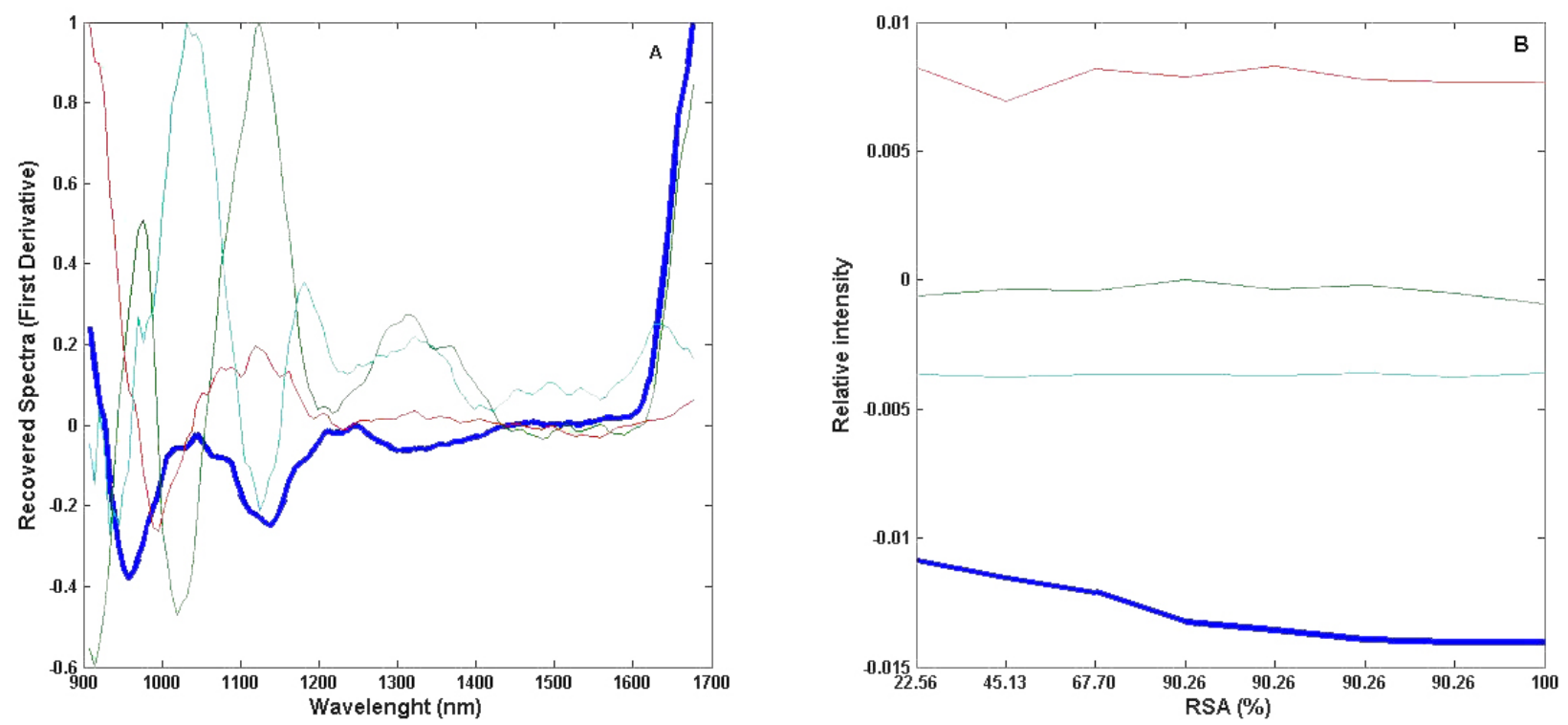

Figure 2: MCR-ALS results. (A) Recovered spectra. (B) Relative intensity: (-) antioxidants, $(-)$ interferent $1,(-)$ interferent 2, (-) interferent 3.

Kasemsumran et al. [25,26], in works from turmeric powder, observed absorptions in the NIR range from 1100 to $1620 \mathrm{~nm}$, mainly due to the $\mathrm{R}-\mathrm{OH}$ bonds and $\mathrm{CH}$ absorptions, similar to that identified in this work. Jovanovic et al. [27], reported that the mechanism of antioxidant activity from curcumin (present in higher concentrations among curcuminoids) is through the donation of hydrogen from the methylene $\mathrm{CH}$ subunit at acid $\mathrm{pH}$, which makes these bonds weakened by facilitating the mechanism of donation of $\mathrm{H}$, in addition to the predicted antioxidant activity related to phenolic hydroxyls [28], suggesting that the $\mathrm{pH}$ used in this work to extract the curcuminoids helped to obtain the antioxidant activity from them.

The relative intensity related to antioxidants were plotted against \%RSA result in a pseudo-univariate calibration model, as shown in Figure 3, with correlation coefficient 0.9532 . By using this model, the $\%$ RSA determination in four independent samples (Table I) with mean \%RSA (obtained by the reference method) of $90.26 \%$ shows a mean $\%$ RSA of $87.5 \%$.

Table I. Results obtained from reference method and by pseudounivariate calibration curve for four independent samples

\begin{tabular}{cc}
\hline $\begin{array}{c}\text { \%SA } \\
\text { Reference method }\end{array}$ & $\begin{array}{c}\text { \%RSA } \\
\text { Pseudo-univariate calibration }\end{array}$ \\
\hline 90.26 & 87.5 \\
90.25 & 87.5 \\
91.95 & 90.0 \\
88.57 & 85.0 \\
Mean $=90.26$ & Mean $=87.5$ \\
\hline
\end{tabular}




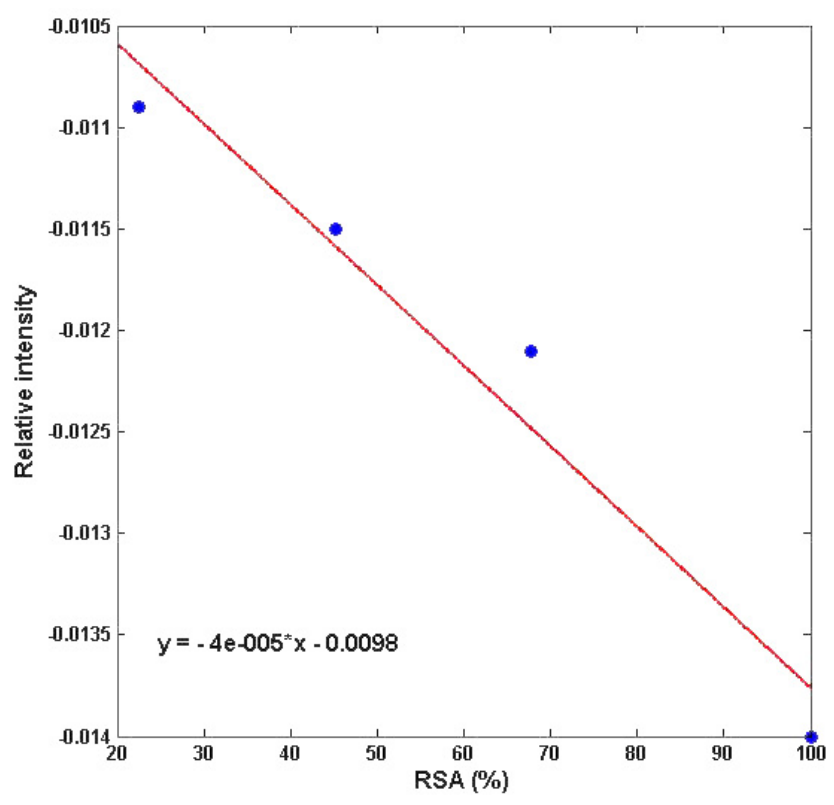

Figure 3. Pseudo-univariate calibration curve.

The results obtained in this proposal show the possibility of \%RSA determination directly from NIR spectra of turmeric powder extracts by using the multivariate resolution method MCR-ALS. In summary, the steps to perform this method are: 1) extraction of curcuminoids (with the ethanol concentration $70 \%$, extraction time 15 minutes, and with drying oven process); 2) prepare the samples; 3) obtaining the NIR spectra; 4) performer MCR-ALS; 5) prepare the pseudo-univariate calibration curve; 6) test the samples with unknown concentration.

\section{CONCLUSION}

Pseudo-univariate calibration model based on MCR-ALS shows the possibility of antioxidant activity determination in extracts from turmeric powder directly from NIR spectra. The main advantage in this alternative method is that the DPPH reagent is not necessary (when the pseudo-univariate curve is ready), which is important in terms of reagents consuming, and waste generation.

Manuscript submitted: Nov. 26, 2018; revised manuscript submitted: Feb. 26, 2019; manuscript accepted: March 25, 2019; published online: June 11, 2019.

\section{REFERENCES}

1. Senise, P. Quim. Nova, 1983, 6, pp 112-116.

2. Laczkowski, M. S.; Gonçalves, T. R.; Gomes, S. T. M.; Março, P. H.; Valderrama, P.; Matsushita, M. LWT - Food Sci. Technol., 2018, 95, pp 303-307.

3. Decker, E. A. Nutr. Rev., 1997, 55, pp 396-398.

4. Deng, J.; Cheng, W.; Yang, G. Food Chem., 2011, 125, pp 1430-1435.

5. Moon, J. -K.; Shibamoto, T. J. Agric. Food Chem., 2009, 57, pp 1655-1666.

6. Scherer, R.; Godoy, H. T. Food Chem., 2009, 112, pp 654-658.

7. Duarte-Almeida, J. M.; Santos, R. J.; Genovese, M. I.; Lajolo, F. M. Ciênc. Tecnol. Aliment., 2006, 26, pp 446-452.

8. Ferreira, S. L. C.; dos Santos, W. N. L.; Bezerra, M. A.; Lemos, V. A.; Bosque-Sendra, J. M. Anal. 
Bioanal. Chem., 2003, 375, pp 443-449.

9. Ribeiro, G. M.; Madivadua, D. A.; Curti, S. M. M.; Pantean, L. P.; Março, P. H.; Valderrama, P. Microchem. J., 2017, 134, pp 114-118.

10. Mamián-López, M.; Poppi, R. J. Anal. Chim. Acta, 2013, 760, pp 53-59.

11. Março, P. H.; Valderrama, P.; Alexandrino, G. L.; Poppi, R. J.; Tauler, R. Quim. Nova, 2014, 37, pp 1525-1532.

12. de Juan, A.; Tauler, R. Crit. Rev. Anal. Chem., 2006, 36, pp 163-176.

13. Parastar, H.; Tauler, R. Anal. Chem., 2014, 86, pp 286-297.

14. Vieira, J. A. G.; Jorge, N. Aliment. Nutr., 1997, 8, pp 39-47.

15. Gopal, J.; Muthu, M.; Chun, S. -C. RSC Adv., 2015, 5, pp 48391-48398.

16. Paulucci, V. P.; Couto, R. O.; Teixeira, C. C. C.; Freitas, L. A. P. Braz. J. Pharmacogn., 2013, 23, pp 94-100.

17. Rouhani, S.; Alizadeh, N.; Salimi, S.; Haji-Ghasemi, T. Prog. Color Colorants Coat., 2009, 2, pp 103-113.

18. Brand-Williams, W.; Cuvelier, M. E.; Berset, C. LWT - Food Sci. Technol., 1995, 28, pp 25-30.

19. Rodrigues-Brandão, I.; Kleinowski, A. M.; Einhardt, A. M.; Lima, M. C.; Amarante, L.; Peters, J. A.; Braga, E. J. B. Cienc. Rural, 2014, 44, pp 1893-1898.

20. Cheng, Z.; Moore, J.; Yu, L. J. Agric. Food Chem., 2006, 54, pp 7429-7436.

21. Jaumot, J.; de Juan, A.; Tauler, R. Chemom. Intell. Lab. Syst., 2015, 140, pp 1-12.

22. Savitzky, A.; Golay, M. J. E. Anal. Chem., 1964, 36, pp 1627-1639.

23. Sabin, G. P.; Lozano, V. A.; Rocha, W. F. C.; Romão, W.; Ortiz, R. S.; Poppi, R. J. J. Pharm. Biom. Anal., 2013, 85, pp 207-212.

24. Burns, D. A.; Ciurczak, E. W. Handbook of near-infrared analysis, $3^{\text {rd }}$ ed. CRC Press, Boca Raton, 2008.

25. Kasemsumran, S.; Apiwatanapiwat, W.; Suttiwijitpukdee, N.; Vaithanomsat, P.; Thanapase, W. J. Near Infrared Spectrosc., 2014, 22, pp 113-120.

26. Kasemsumran, S.; Suttiwijitpukdee, N.; Keeratinijakal, V. Anal. Sci., 2017, 33, pp 111-115.

27. Jovanovic, S. V.; Steenken, S.; Boone, C. W.; Simic, M. G. J. Am. Chem. Soc., 1999, 121, pp 9677-9681.

28. Ak, T.; Gülçin, I. Chem.-Biol. Interac., 2008, 174, pp 27-37. 